\title{
Article
}

\section{Anti complex fuzzy subgroups under s-norms}

\author{
Rasul Rasuli \\ Mathematics Department, Faculty of Science Payame Noor University(PNU), Tehran, Iran.; rasulirasul@yahoo.com \\ Received: 11 July 2020; Accepted: 25 September 2020; Published: 20 October 2020.
}

\begin{abstract}
In this study, we define anti complex fuzzy subgroups and normal anti complex fuzzy subgroups under s-norms and investigate some of characteristics of them. Later we introduce and study the intersection and composition of them. Next, we define the concept normality between two anti complex fuzzy subgroups by using s-norms and obtain some properties of them. Finally, we define the image and the inverse image of them under group homomorphisms.
\end{abstract}

Keywords: Group theory, fuzzy groups, norms, intersections, compositions, complex fuzzy subgroups, normal complex fuzzy subgroups.

\section{Introduction}

G roup theory has applications in physics, chemistry, and computer science, and even puzzles like Rubik's Cube can be represented using group theory. Fuzzy sets, proposed by Zadeh [1], are sets whose elements have degrees of membership. Rosenfeld [2] introduced fuzzy sets in the realm of group theory and formulated the concepts of fuzzy subgroups of a group. Many authors have worked on fuzzy group theory [2-4], especially, some authors considered the fuzzy subgroups with respect to norms [5-7]. Alsarahead and Ahmad [8] defined the complex fuzzy subgroup and investigate some of its characteristics. The author by using norms, investigated some properties of fuzzy algebraic structures [9-11].

In this paper, by using s-norms, we define and investigate some properties of anti complex fuzzy subgroups of group $G$ under s-norm $S$ as $A C F S(G)$. Also we define the composition and intersection of two $\mu_{1}, \mu_{2} \in A C F S(G)$ and obtain some of their characteristics. Later, we introduce and investigate the normality of $\mu \in A C F S(G)$ denoted by NACFS $(G)$. Finally, we introduce the normality between two $\mu_{1}, \mu_{2} \in A C F S(G)$ as $\mu_{1} \bowtie \mu_{2}$ and investigate some important properties of them. By using a group homomorphism $f: G \rightarrow H$, we prove that if $\mu \in \operatorname{ACFS}(G)$ and $v \in \operatorname{ACFS}(H)$, then $f(\mu) \in \operatorname{ACFS}(H)$ and $f^{-1}(v) \in \operatorname{ACFS}(G)$. Also if $\mu \in N A C F S(G)$ and $v \in N A C F S(H)$, then we prove that $f(\mu) \in N A C F S(H)$ and $f^{-1}(v) \in N A C F S(G)$. Also we show that if $\mu_{1}, \mu_{2} \in A C F S(G)$ such that $\mu_{1} \bowtie \mu_{2}$, then we show that $f\left(\mu_{1}\right) \bowtie f\left(\mu_{2}\right)$ and if $\mu_{1}, \mu_{2} \in \operatorname{ACFS}(H)$ such that $\mu_{1} \bowtie \mu_{2}$, then we obtain $f^{-1}\left(\mu_{1}\right) \bowtie f^{-1}\left(\mu_{2}\right)$.

\section{Preliminaries}

The following definitions and preliminaries are required in the sequel of our work and hence presented in brief. For details we refer readers to [6,12-15].

Definition 1. A group is a non-empty set $G$, on which there is a binary operation $(a, b) \rightarrow a b$ such that

- if $a$ and $b$ belong to $G$ then ab is also in $G$ (closure),

- $a(b c)=(a b) c$ for all $a, b, c \in G$ (associativity),

- there is an element $e_{G} \in G$ such that $a e_{G}=e e_{G} a=a$ for all $a \in G$ (identity),

- if $a \in G$, then there is an element $a^{-1} \in G$ such that $a a^{-1}=a^{-1} a=e_{G}$ (inverse).

One can easily check that this implies the unicity of the identity and of the inverse. A group $G$ is called abelian if the binary operation is commutative, i.e., $a b=b a$ for all $a, b \in G$.

Remark 1. There are two standard notations for the binary group operation: either the additive notation, that is $(a, b) \rightarrow a+b$ in which case the identity is denoted by 0 , or the multiplicative notation, that is $(a, b) \rightarrow a b$ for which the identity is denoted by $e$. 
Definition 2. Let $G$ be an arbitrary group with a multiplicative binary operation and identity $e$. By a fuzzy subset of $G$, we mean a function from $G$ into $[0,1]$. The set of all fuzzy subsets of $G$ is called the $[0,1]$-power set of $G$ and is denoted $[0,1]^{G}$.

Definition 3. Let $X$ be a nonempty set. A complex fuzzy set $A$ on $X$ is an object having the form $A=$ $\left\{\left(x, \mu_{A}(x)\right) \mid x \in X\right\}$, where $\mu_{A}$ denotes the degree of membership function that assigns each element $x \in X, \mathrm{a}$ complex number $\left.\mu_{A}(x)\right)$ lies within the unit circle in the complex plane. We shall assume that $\mu_{A}(x)$ will be represented by $r_{A}(x) e^{i w_{A}(x)}$, where $i=\sqrt{-1}$, and $r: X \rightarrow[0,1]$ and $w: X \rightarrow[0,2 \pi]$. Note that by setting $w(x)=0$ in the definition above, we return back to the traditional fuzzy subset. Let $\mu_{1}=r_{1} e^{w_{1}}$ and $\mu_{2}=r_{2} e^{w_{2}}$ be two complex numbers lie within the unit circle in the complex plane. By $\mu_{1} \leq \mu_{2}$, we mean $r_{1} \leq r_{2}$ and $w_{1} \leq w_{2}$.

Definition 4. An $s$-norm $S$ is a function $S:[0,1] \times[0,1] \rightarrow[0,1]$ having the following four properties:

- $S(x, 0)=x$,

- $S(x, y) \leq S(x, z)$ if $y \leq z$,

- $S(x, y)=S(y, x)$,

- $S(x, S(y, z))=S(S(x, y), z)$

for all $x, y, z \in[0,1]$.

We say that $S$ is idempotent if for all $x \in[0,1], S(x, x)=x$.

Example 1. The basic s-norms are $S_{m}(x, y)=\max \{x, y\}, S_{b}(x, y)=\min \{1, x+y\}$ and $S_{p}(x, y)=x+y-x y$ for all $x, y \in[0,1] . S_{m}$ is standard union, $S_{b}$ is bounded sum, $S_{p}$ is algebraic sum.

Lemma 1. Let $S$ be an s-norm. Then

$$
S(S(x, y), S(w, z))=S(S(x, w), S(y, z))
$$

for all $x, y, w, z \in[0,1]$.

\section{Anti complex fuzzy subgroups under s-norms}

Definition 5. Let $G$ be a group and $\mu: G \rightarrow[0,1]$ be a complex fuzzy set on $G$. Then $\mu=r e^{i w}$ is said to be an anti complex fuzzy subgroup of $G$ under s-norm $S$ if the following conditions hold:

- $r(x y) \leq S(r(x), r(y))$,

- $r\left(x^{-1}\right) \leq r(x)$,

- $w(x y) \leq \max \{w(x), w(y)\}$,

- $w\left(x^{-1}\right) \leq w(x)$,

for all $x, y \in G$. The set of all anti complex fuzzy subgroups of $G$ under s-norm $S$ is denoted by $A C F S(G)$.

Example 2. Let $G=\{0, a, b, c\}$ be the Klein's group. Every element is its own inverse, and the product of any two distinct non-identity elements is the remaining non-identity element. Thus the Klein 4-group admits the elegant presentation $a^{2}=b^{2}=c^{2}=a b c=0$. Define $r: G \rightarrow[0,1]$ by

$$
r(x)= \begin{cases}0.35 & \text { if } x=a \\ 0.45 & \text { if } x=b \\ 0.65 & \text { if } x=c \\ 0.85 & \text { if } x=0\end{cases}
$$


and $w: G \rightarrow[0,2 \pi]$ by

$$
w(x)= \begin{cases}0.4 \pi & \text { if } x=a \\ 0.4 \pi & \text { if } x=b \\ 0.5 \pi & \text { if } x=c \\ 0.6 \pi & \text { if } x=0 .\end{cases}
$$

Let $S(a, b)=S_{p}(a, b)=a+b-a b$ for all $a, b \in[0,1]$, then $\mu(x)=r(x) e^{i w(x)} \in \operatorname{ACFS}(G)$ for all $x \in G$.

Proposition 1. Let $\mu=r e^{i w} \in A C F S(G)$ such that $C$ be idempotent s-norm. Then

- $\mu(e) \leq \mu(x)$ for all $x \in G$,

- $\mu\left(x^{n}\right) \leq \mu(x)$ for all $x \in G$ and $n \geq 1$,

- $\mu(x)=\mu\left(x^{-1}\right)$ for all $x \in G$.

Proof. Let $\mu=r e^{i w} \in \operatorname{ACFS}(G)$ and $x \in G$ and $n \geq 1$. Then

$$
\begin{aligned}
(1) r(e) & =r\left(x x^{-1}\right) \leq S\left(r(x), r\left(x^{-1}\right)\right) \leq S(r(x), r(x))=r(x), \\
w(e) & =w\left(x x^{-1}\right) \leq \max \left\{w(x), w\left(x^{-1}\right)\right\} \leq \max \{w(x), w(x)\}=w(x), \\
\mu(e) & =r(e) e^{i w(x)} \leq r(x) e^{i w(x)}=\mu(x), \\
r\left(x^{n}\right) & =r(\underbrace{x x \ldots x}_{n}) \leq S(\underbrace{r(x), r(x), \ldots, r(x)}_{n})=r(x), \\
w\left(x^{n}\right) & =w(\underbrace{x x \ldots x}_{n}) \leq \max \{\underbrace{r(x), r(x), \ldots, r(x)}_{n}\}=w(x), \\
\mu\left(x^{n}\right) & =r\left(x^{n}\right) e^{i w\left(x^{n}\right)} \leq r(x) e^{i w(x)}=\mu(x), \\
r(x) & =r\left(\left(x^{-1}\right)\right)^{-1} \leq r\left(x^{-1}\right) \leq r(x), \\
r(x) & =r\left(x^{-1}\right), \\
w(x) & =w\left(\left(x^{-1}\right)\right)^{-1} \leq w\left(x^{-1}\right) \leq w(x), \\
w(x) & =w\left(x^{-1}\right), \\
\mu(x) & =r(x) e^{i w(x)}=r\left(x^{-1}\right) e^{i w\left(x^{-1}\right)}=\mu\left(x^{-1}\right) .
\end{aligned}
$$

Proposition 2. Let $\mu=r e^{i w} \in A C F S(G)$ and $x \in G$ such that $S$ be idempotent s-norm. Then $\mu(x y)=\mu(y) \forall y \in G$ if and only if $\mu(x)=\mu(e)$.

Proof. Let $\mu(x y)=\mu(y) \forall y \in G$. As $y=e$, so $\mu(x)=\mu(e)$. Conversely, let $\mu(x)=\mu(e)$, then $r(x)=r(e)$ and $w(x)=w(e)$. From Proposition 1, we get $r(x) \leq r(y)$ and $r(x) \leq r(x y)$. Also $w(x) \leq w(y)$ and $w(x) \leq w(x y)$. Now $r(x y) \leq S(r(x), r(y)) \leq S(r(y), r(y))=r(y)=r\left(x^{-1} x y\right) \leq S(r(x), r(x y)) \leq S(r(x y), r(x y))=r(x y)$.

Also $w(x y) \leq \max \{w(x), w(y)\} \leq \max \{w(y), w(y)\}=w(y)=w\left(x^{-1} x y\right) \leq \max \{w(x), w(x y)\} \leq$ $\max \{w(x y), w(x y)\}=w(x y)$. Therefore $\mu(x y)=r(x y) e^{i w(x y)}=r(y) e^{i w(y)}=\mu(y)$.

Definition 6. Let $G$ be a set and $\mu_{1}=r_{1} e^{i w_{1}}, \mu_{2}=r_{2} e^{i w_{2}}$ be two complex fuzzy sets on $G$. Denote the composition of $\mu_{1}$ and $\mu_{2}$ as $\mu_{1} \circ \mu_{2}=\left(r_{1} \circ r_{2}\right) e^{i\left(w_{1} \circ w_{2}\right)}$ such that $r_{1} \circ r_{2}: G \rightarrow[0,1]$ and $w_{1} \circ w_{2}: G \rightarrow[0,2 \pi]$ and define by $\left(\mu_{1} \circ \mu_{2}\right)(x)=\left(r_{1} \circ r_{2}\right)(x) e^{i\left(w_{1} \circ w_{2}\right)(x)}(x)$ such that

$$
\left(r_{1} \circ r_{2}\right)(x)=\left\{\begin{aligned}
\inf _{x=a b} S\left(r_{1}(a), r_{2}(b)\right) & \text { if } x=a b \\
0 & \text { if } x \neq a b
\end{aligned}\right.
$$

and

$$
\left(w_{1} \circ w_{2}\right)(x)=\left\{\begin{aligned}
\max _{x=a b}\left\{w_{1}(a), w_{2}(b)\right\} & \text { if } x=a b, \\
0 & \text { if } x \neq a b,
\end{aligned}\right.
$$

thus $\left(\mu_{1} \circ \mu_{2}\right)(x)=\inf _{x=a b} S\left(r_{1}(a), r_{2}(b)\right) e \cdot i \max _{x=a b}\left\{w_{1}(a), w_{2}(b)\right\}$. 
Proposition 3. Let $\mu^{-1}$ be the inverse of $\mu$ such that $\mu^{-1}(x)=\mu\left(x^{-1}\right)$. Then $\mu \in A C F S(G)$ if and only if $\mu$ satisfies the following conditions:

(1) $\mu \leq \mu \circ \mu$;

(2) $\mu^{-1}=\mu$.

Proof. Let $x, y, z \in G$ with $x=y z$ and $\mu \in \operatorname{ACFS}(G)$. Then

$$
\begin{aligned}
r(x) & =r(y z) \leq S(r(y), r(z))=(r \circ r)(x), \\
w(x) & =w(y z) \leq \max \{r(y), r(z)\}=(w \circ w)(x), \\
\mu(x) & =r(x) e^{i w(x)} \leq(r \circ r)(x) e^{i(w \circ w)(x)}=(\mu \circ \mu)(x),
\end{aligned}
$$

so $\mu \leq \mu \circ \mu$.

Also from Proposition 1, for all $x \in G$, we have that $\mu^{-1}(x)=\mu\left(x^{-1}\right)=\mu(x)$ and so $\mu^{-1}=\mu$. Conversely let $\mu \leq \mu \circ \mu$ and $\mu^{-1}=\mu$. We prove that $\mu \in \operatorname{ACFS}(G)$. As $\mu \leq \mu \circ \mu$ so $r(x) \leq(r \circ r)(x)$ and $w(x) \leq$ $(w \circ w)(x)$. Thus

$$
r(y z)=r(x) \leq(r \circ r)(x)=\inf _{x=y z} S(r(y), r(z)) \leq S(r(y), r(z))
$$

and

$$
w(y z)=w(x) \geq(w \circ w)(x)=\max _{x=y z}\{w(y), w(z)\} \geq\{w(y), w(z)\} .
$$

Since $\mu^{-1}=\mu$ so $r^{-1}(x)=r(x)$ and $w^{-1}(x)=w(x)$. Therefore $r\left(x^{-1}\right)=r^{-1}(x)=r(x)$ and $w\left(x^{-1}\right)=$ $w^{-1}(x)=w(x)$. Then $\mu \in \operatorname{ACFS}(G)$.

Corollary 1. Let $\mu_{1}, \mu_{2} \in A C F S(G)$ and $G$ be commutative group. Then $\mu_{1} \circ \mu_{2} \in A C F S(G)$ if and only if $\mu_{1} \circ \mu_{2}=$ $\mu_{2} \circ \mu_{1}$.

Proof. As $\mu_{1}, \mu_{2} \in \operatorname{CFST}(G)$ and $\mu_{1} \circ \mu_{2} \in \operatorname{ACFS}(G)$, so from Proposition 3, we get $\mu_{1}^{-1}=\mu_{1}$ and $\mu_{2}^{-1}=\mu_{2}$ and $\left(\mu_{2} \circ \mu_{1}\right)^{-1}=\mu_{2} \circ \mu_{1}$. Then $\mu_{1} \circ \mu_{2}=\mu_{1}^{-1} \circ \mu_{2}^{-1}=\left(\mu_{2} \circ \mu_{1}\right)^{-1}=\mu_{2} \circ \mu_{1}$. Conversely, let $\mu_{1} \circ \mu_{2}=\mu_{2} \circ \mu_{1}$, then $\left(\mu_{1} \circ \mu_{2}\right) \circ\left(\mu_{1} \circ \mu_{2}\right)=\mu_{1} \circ\left(\mu_{2} \circ \mu_{1}\right) \circ \mu_{2}=\mu_{1} \circ\left(\mu_{1} \circ \mu_{2}\right) \circ \mu_{2}=\left(\mu_{1} \circ \mu_{1}\right) \circ\left(\mu_{2} \circ \mu_{2}\right) \geq \mu_{1} \circ \mu_{2}$. Also $\left(\mu_{1} \circ \mu_{2}\right)^{-1}=\left(\mu_{2} \circ \mu_{1}\right)^{-1}=\mu_{1}^{-1} \circ \mu_{2}^{-1}=\mu_{1} \circ \mu_{2}$. Then the Proposition 3 gives us that $\mu_{1} \circ \mu_{2} \in A C F S(G)$.

Definition 7. Let $\mu_{1}=r_{1} e^{i w_{1}} \in A C F S(G)$ and $\mu_{2}=r_{2} e^{i w_{2}} \in A C F S(G)$. Define the intersection $\mu_{1} \cap \mu_{2}$ as $\mu_{1} \cap \mu_{2}=r_{1} e^{i w_{1}} \cap r_{2} e^{i w_{2}}=\left(r_{1} \cap r_{2}\right) e^{i\left(w_{1} \cap w_{2}\right)}$ such that $r_{1} \cap r_{2}: G \rightarrow[0,1]$ and $w_{1} \cap w_{2}: G \rightarrow[0,2 \pi]$ and for all $x \in G$, define $\left(r_{1} \cap r_{2}\right)(x)=S\left(r_{1}(x), r_{2}(x)\right)$ and $\left(w_{1} \cap r_{2}\right)(x)=\max \left\{w_{1}(x), w_{2}(x)\right\}$.

Proposition 4. Let $\mu_{1}=r_{1} e^{i w_{1}} \in \operatorname{ACFS}(G)$ and $\mu_{2}=r_{2} e^{i w_{2}} \in \operatorname{ACFS}(G)$. Then $\mu_{1} \cap \mu_{2} \in \operatorname{ACFS}(G)$.

Proof. (1) Let $g_{1}, g_{2} \in G$. Then

$$
\begin{aligned}
\left(r_{1} \cap r_{2}\right)\left(g_{1} g_{2}\right) & =S\left(r_{1}\left(g_{1} g_{2}\right), r_{2}\left(g_{1} g_{2}\right)\right) \leq S\left(S\left(r_{1}\left(g_{1}\right), r_{1}\left(g_{2}\right)\right), S\left(r_{2}\left(g_{1}\right), r_{2}\left(g_{2}\right)\right)\right) \\
& =S\left(S\left(r_{1}\left(g_{1}\right), r_{2}\left(g_{1}\right)\right), T\left(r_{1}\left(g_{2}\right), r_{2}\left(g_{2}\right)\right)\right) \\
& =S\left(\left(r_{1} \cap r_{2}\right)\left(g_{1}\right),\left(r_{1} \cap r_{2}\right)\left(g_{2}\right)\right),
\end{aligned}
$$

and thus $\left(r_{1} \cap r_{2}\right)\left(g_{1} g_{2}\right) \leq S\left(\left(r_{1} \cap r_{2}\right)\left(g_{1}\right),\left(r_{1} \cap r_{2}\right)\left(g_{2}\right)\right)$.

(2) If $g \in G$, then

$$
\begin{aligned}
\left(r_{1} \cap r_{2}\right)\left(g^{-1}\right)=S\left(r_{1}\left(g^{-1}\right), r_{2}\left(g^{-1}\right)\right) & \leq S\left(r_{1}(g), r_{2}(g)\right)=\left(r_{1} \cap r_{2}\right)(g) \\
\left(r_{1} \cap r_{2}\right)\left(g^{-1}\right) & \geq\left(r_{1} \cap r_{2}\right)(g) .
\end{aligned}
$$


(3) Let $g_{1}, g_{2} \in G$. Then

$$
\begin{aligned}
\left(w_{1} \cap w_{2}\right)\left(g_{1} g_{2}\right) & =\max \left\{w_{1}\left(g_{1} g_{2}\right), w_{2}\left(g_{1} g_{2}\right)\right\} \\
& \leq \max \left\{\max \left\{w_{1}\left(g_{1}\right), w_{1}\left(g_{2}\right)\right\}, \max \left\{w_{2}\left(g_{1}\right), w_{2}\left(g_{2}\right)\right\}\right\} \\
& =\max \left\{\max \left\{w_{1}\left(g_{1}\right), w_{2}\left(g_{1}\right)\right\}, \max \left\{w_{1}\left(g_{2}\right), w_{2}\left(g_{2}\right)\right)\right\} \\
& =\max \left\{\left(w_{1} \cap w_{2}\right)\left(g_{1}\right),\left(w_{1} \cap w_{2}\right)\left(g_{2}\right)\right) \\
\left(w_{1} \cap w_{2}\right)\left(g_{1} g_{2}\right) & \leq \max \left\{\left(w_{1} \cap w_{2}\right)\left(g_{1}\right),\left(w_{1} \cap w_{2}\right)\left(g_{2}\right)\right) .
\end{aligned}
$$

(4) Let $g \in G$ so

$$
\begin{aligned}
\left(w_{1} \cap w_{2}\right)\left(g^{-1}\right) & =\max \left\{w_{1}\left(g^{-1}\right), w_{21}\left(g^{-1}\right)\right\} \\
& \leq \max \left\{w_{1}(g), w_{2}(g)\right\} \\
& =\left(w_{1} \cap w_{2}\right)(g) \\
\left(w_{1} \cap w_{2}\right)\left(g^{-1}\right) & \leq\left(w_{1} \cap w_{2}\right)(g) .
\end{aligned}
$$

Thus from (1)-(4) we give that $\mu_{1} \cap \mu_{2} \in \operatorname{ACFS}(G)$.

Corollary 2. Let $I_{n}=\{1,2, \ldots, n\}$. If $\left\{\mu_{i} \mid i \in I_{n}\right\} \subseteq \operatorname{ACFS}(G)$ then $\mu=\cap_{i \in I_{n}} \mu_{i} \in \operatorname{ACFS}(G)$.

Definition 8. $\mu \in \operatorname{ACFS}(G)$ is called normal if for all $x, y \in G$ we have that $\mu\left(x y x^{-1}\right)=\mu(y)$. The set of all normal anti complex fuzzy subgroups of $G$ under s-norm $S$ ia denoted by NACFS(G).

Proposition 5. Let $\mu_{1}=r_{1} e^{i w_{1}} \in N A C F S(G)$ and $\mu_{2}=r_{2} e^{i w_{2}} \in N A C F S(G)$. Then $\mu_{1} \cap \mu_{2} \in \operatorname{NACFS}(G)$.

Proof. From Proposition 4 we will have that $\mu_{1} \cap \mu_{2} \in A C F S(G)$. Let $g_{1}, g_{2} \in G$ then

$$
\begin{aligned}
\left(r_{1} \cap r_{2}\right)\left(g_{1} g_{2} g_{1}^{-1}\right) & =S\left(r_{1}\left(g_{1} g_{2} g_{1}^{-1}\right), r_{2}\left(g_{1} g_{2} g_{1}^{-1}\right)\right)=S\left(r_{1}\left(g_{2}\right), r_{2}\left(g_{2}\right)\right)=\left(r_{1} \cap r_{2}\right)\left(g_{2}\right) \\
\left(w_{1} \cap w_{2}\right)\left(g_{1} g_{2} g_{1}^{-1}\right) & =\max \left\{w_{1}\left(g_{1} g_{2} g_{1}^{-1}\right), w_{2}\left(g_{1} g_{2} g_{1}^{-1}\right)\right\}=\max \left\{w_{1}\left(g_{2}\right), w_{2}\left(g_{2}\right)\right\}=\left(w_{1} \cap w_{2}\right)\left(g_{2}\right) \\
\left(\mu_{1} \cap \mu_{2}\right)\left(g_{1} g_{2} g_{1}^{-1}\right) & =\left(r_{1} \cap r_{2}\right)\left(g_{1} g_{2} g_{1}^{-1}\right) e^{i\left(w_{1} \cap w_{2}\right)\left(g_{1} g_{2} g_{1}^{-1}\right)}=\left(r_{1} \cap r_{2}\right)\left(g_{2}\right) e^{i\left(w_{1} \cap w_{2}\right)\left(g_{2}\right)}=\left(\mu_{1} \cap \mu_{2}\right)\left(g_{2}\right)
\end{aligned}
$$

and therefore $\mu_{1} \cap \mu_{2} \in \operatorname{NACFS}(G)$.

Corollary 3. Let $I_{n}=\{1,2, \ldots, n\}$. If $\left\{\mu_{i} \mid i \in I_{n}\right\} \subseteq \operatorname{NACFS}(G)$, then $\mu=\cap_{i \in I_{n}} \mu_{i} \in \operatorname{NACFS}(G)$.

Definition 9. Let $\mu_{1}=r_{1} e^{i w_{1}} \in \operatorname{ACFS}(G)$ and $\mu_{2}=r_{2} e^{i w_{2}} \in \operatorname{ACFS}(G)$ such that $\mu_{1} \subseteq \mu_{2}$. We say that $\mu_{1}$ is normal of the $\mu_{2}$, written $\mu_{1} \bowtie \mu_{2}$, if $r_{1}\left(g_{1} g_{2} g_{1}^{-1}\right) \leq S\left(r_{1}\left(g_{2}\right), r_{2}\left(g_{1}\right)\right)$ and $w_{1}\left(g_{1} g_{2} g_{1}^{-1}\right) \leq \max \left\{w_{1}\left(g_{2}\right), w_{2}\left(g_{1}\right)\right\}$ for all $g_{1}, g_{2} \in G$.

Proposition 6. If $S$ be idempotent s-norm, then every $\mu=r e^{i w} \in A C F S(G)$ will be normal of itself.

Proof. Let $g_{1}, g_{2} \in G$ and $\mu=r e^{i w} \in A C F S(G)$, then

$$
\begin{aligned}
r\left(g_{1} g_{2} g_{1}^{-1}\right) & \leq S\left(r\left(g_{1}\right), r\left(g_{2} g_{1}^{-1}\right)\right) \\
& \leq S\left(r\left(g_{1}\right), S\left(r\left(g_{2}\right), r\left(g_{1}^{-1}\right)\right)\right) \\
& \leq S\left(r\left(g_{1}\right), S\left(r\left(g_{2}\right), r\left(g_{1}\right)\right)\right) \\
& =S\left(r\left(g_{2}\right), S\left(r\left(g_{1}\right), r\left(g_{1}\right)\right)\right) \\
& =S\left(r\left(g_{2}\right), r\left(g_{1}\right)\right) \\
\left(g_{1} g_{2} g_{1}^{-1}\right) & \leq S\left(r\left(g_{2}\right), r\left(g_{1}\right)\right) .
\end{aligned}
$$


Also

$$
\begin{aligned}
w\left(g_{1} g_{2} g_{1}^{-1}\right) & \left.\leq \max \left\{w\left(g_{1}\right), w\left(g_{2} g_{1}^{-1}\right)\right)\right\} \\
& \leq \max \left\{w\left(g_{1}\right), \max \left\{w\left(g_{2}\right), w\left(g_{1}^{-1}\right)\right\}\right\} \\
& \leq \max \left\{w\left(g_{1}\right), \max \left\{w\left(g_{2}\right), w\left(g_{1}\right)\right)\right) \\
& =\max \left\{w\left(g_{2}\right), \max \left\{w\left(g_{1}\right), w\left(g_{1}\right)\right\}\right\} \\
& =\max \left\{w\left(g_{2}\right), w\left(g_{1}\right)\right\} \\
w\left(g_{1} g_{2} g_{1}^{-1}\right) & \leq \max \left\{w\left(g_{2}\right), w\left(g_{1}\right)\right\}
\end{aligned}
$$

Therefore $\mu=r e^{i w} \bowtie \mu=r e^{i w}$.

Proposition 7. Let $\mu_{1}=r_{1} e^{i w_{1}} \in N A C F S(G)$ and $\mu_{2}=r_{2} e^{i w_{2}} \in A C F S(G)$ such that S be idempotent s-norm, then $\mu_{1} \cap \mu_{2} \bowtie \mu_{2}$.

Proof. As Proposition $4\left(\mu_{1} \cap \mu_{2}\right) \leq \mu_{2}$ and $\left(\mu_{1} \cap \mu_{2}\right) \in \operatorname{ACFS}(G)$. Let $g_{1}, g_{2} \in G$ and $\mu_{1} \cap \mu_{2}=\left(r_{1} \cap\right.$ $\left.r_{2}\right) e^{i\left(w_{1} \cap w_{2}\right)}$, then

$$
\begin{aligned}
\left(r_{1} \cap r_{2}\right)\left(g_{1} g_{2} g_{1}^{-1}\right) & =S\left(r_{1}\left(g_{1} g_{2} g_{1}^{-1}\right), r_{2}\left(g_{1} g_{2} g_{1}^{-1}\right)\right) \\
& =S\left(r_{1}\left(g_{2}\right), r_{2}\left(g_{1} g_{2} g_{1}^{-1}\right)\right) \\
& \leq S\left(r_{1}\left(g_{2}\right), S\left(r_{2}\left(g_{1} g_{2}\right), r_{2}\left(g_{1}^{-1}\right)\right)\right) \\
& \leq S\left(r_{1}\left(g_{2}\right), S\left(r_{2}\left(g_{1} g_{2}\right), r_{2}\left(g_{1}\right)\right)\right) \\
& \leq S\left(r_{1}\left(g_{2}\right), S\left(S\left(r_{2}\left(g_{1}\right), r_{2}\left(g_{2}\right)\right), r_{2}\left(g_{1}\right)\right)\right) \\
& =S\left(r_{1}\left(g_{2}\right), S\left(S\left(r_{2}\left(g_{1}\right), r_{2}\left(g_{1}\right)\right), r_{2}\left(g_{2}\right)\right)\right) \\
& =S\left(r_{1}\left(g_{2}\right), S\left(r_{2}\left(g_{1}\right), r_{2}\left(g_{2}\right)\right)\right) \\
& =S\left(S\left(r_{1}\left(g_{2}\right), r_{2}\left(g_{2}\right)\right), r_{2}\left(g_{1}\right)\right) \\
& =S\left(\left(r_{1} \cap r_{2}\right)\left(g_{2}\right), r_{2}\left(g_{1}\right)\right),
\end{aligned}
$$

and thus $\left(r_{1} \cap r_{2}\right)\left(g_{1} g_{2} g_{1}^{-1}\right) \leq S\left(\left(r_{1} \cap r_{2}\right)\left(g_{2}\right), r_{2}\left(g_{1}\right)\right)$.

Also

$$
\begin{aligned}
\left(w_{1} \cap w_{2}\right)\left(g_{1} g_{2} g_{1}^{-1}\right) & =\max \left\{w_{1}\left(g_{1} g_{2} g_{1}^{-1}\right), w_{2}\left(g_{1} g_{2} g_{1}^{-1}\right)\right\} \\
& =\max \left\{w_{1}\left(g_{2}\right), w_{2}\left(g_{1} g_{2} g_{1}^{-1}\right)\right\} \\
& \leq \max \left\{w_{1}\left(g_{2}\right), \max \left\{w_{2}\left(g_{1} g_{2}\right), w_{2}\left(g_{1}^{-1}\right)\right\}\right\} \\
& \leq \max \left\{w_{1}\left(g_{2}\right), \max \left\{w_{2}\left(g_{1} g_{2}\right), w_{2}\left(g_{1}\right)\right\}\right\} \\
& \leq \max \left\{w_{1}\left(g_{2}\right), \max \left\{\max \left\{w_{2}\left(g_{1}\right), w_{2}\left(g_{2}\right)\right\}, w_{2}\left(g_{1}\right)\right\}\right\} \\
& \left.=\max \left\{w_{1}\left(g_{2}\right), \max \left\{\max \left\{w_{2}\left(g_{1}\right), w_{2}\left(g_{1}\right)\right)\right\}, w_{2}\left(g_{2}\right)\right\}\right\} \\
& =\max \left\{w_{1}\left(g_{2}\right), \max \left\{w_{2}\left(g_{1}\right), w_{2}\left(g_{2}\right)\right\}\right\} \\
& =\max \left\{\max \left\{w_{1}\left(g_{2}\right), w_{2}\left(g_{2}\right)\right\}, w_{2}\left(g_{1}\right)\right\} \\
& =\max \left\{\left(w_{1} \cap w_{2}\right)\left(g_{2}\right), w_{2}\left(g_{1}\right)\right\},
\end{aligned}
$$

and then $\left(w_{1} \cap w_{2}\right)\left(g_{1} g_{2} g_{1}^{-1}\right) \leq \max \left\{\left(w_{1} \cap w_{2}\right)\left(g_{2}\right), w_{2}\left(g_{1}\right)\right\}$. Therefore $\mu_{1} \cap \mu_{2}=\left(r_{1} \cap r_{2}\right) e^{i\left(w_{1} \cap w_{2}\right)} \bowtie \mu_{2}$.

Proposition 8. Let $\mu_{1}=r_{1} e^{i w_{1}} \in A C F S(G)$ and $\mu_{2}=r_{2} e^{i w_{2}} \in A C F S(G)$ and $\mu_{3}=r_{3} e^{i w_{3}} \in A C F S(G)$ and $S$ be idempotent s-norm. If $\mu_{1} \bowtie \mu_{3}$ and $\mu_{2} \bowtie \mu_{3}$, then $\mu_{1} \cap \mu_{2} \bowtie \mu_{3}$.

Proof. By Proposition 4, we have $\mu_{1} \cap \mu_{2} \in \operatorname{ACFS}(G)$ and $\mu_{1} \cap \mu_{2} \leq \mu_{3}$. Let $g_{1}, g_{2} \in G$. As $\mu_{1} \bowtie \mu_{3}$, so $r_{1}\left(g_{1} g_{2} g_{1}^{-1}\right) \leq S\left(r_{1}\left(g_{2}\right), r_{3}\left(g_{1}\right)\right)$ and $w_{1}\left(g_{1} g_{2} g_{1}^{-1}\right) \leq \max \left\{r_{1}\left(g_{2}\right), r_{3}\left(g_{1}\right)\right\}$ and as $\mu_{2} \bowtie \mu_{3}$ so $r_{2}\left(g_{1} g_{2} g_{1}^{-1}\right) \leq$ $S\left(r_{2}\left(g_{2}\right), r_{3}\left(g_{1}\right)\right)$ and $w_{2}\left(g_{1} g_{2} g_{1}^{-1}\right) \leq \max \left\{w_{2}\left(g_{2}\right), w_{3}\left(g_{1}\right)\right\}$. Now 


$$
\begin{aligned}
\left(r_{1} \cap r_{2}\right)\left(g_{1} g_{2} g_{1}^{-1}\right) & =S\left(r_{1}\left(g_{1} g_{2} g_{1}^{-1}\right), r_{2}\left(g_{1} g_{2} g_{1}^{-1}\right)\right) \\
& \leq S\left(S\left(r_{1}\left(g_{2}\right), r_{3}\left(g_{1}\right)\right), S\left(r_{2}\left(g_{2}\right), r_{3}\left(g_{1}\right)\right)\right) \\
& =S\left(S\left(r_{1}\left(g_{2}\right), r_{2}\left(g_{2}\right)\right), S\left(r_{3}\left(g_{1}\right), r_{3}\left(g_{1}\right)\right)\right) \\
& =S\left(S\left(r_{1}\left(g_{2}\right), r_{2}\left(g_{2}\right)\right), r_{3}\left(g_{1}\right)\right) \\
& =S\left(\left(r_{1} \cap r_{2}\right)\left(g_{2}\right), r_{3}\left(g_{1}\right)\right),
\end{aligned}
$$

and then $\left(r_{1} \cap r_{2}\right)\left(g_{1} g_{2} g_{1}^{-1}\right) \leq S\left(\left(r_{1} \cap r_{2}\right)\left(g_{2}\right), r_{3}\left(g_{1}\right)\right)$. Also

$$
\begin{aligned}
\left(w_{1} \cap w_{2}\right)\left(g_{1} g_{2} g_{1}^{-1}\right) & =\max \left\{w_{1}\left(g_{1} g_{2} g_{1}^{-1}\right), w_{2}\left(g_{1} g_{2} g_{1}^{-1}\right)\right\} \\
& \geq \max \left\{\max \left\{w_{1}\left(g_{2}\right), w_{3}\left(g_{1}\right)\right\}, \max \left\{w_{2}\left(g_{2}\right), w_{3}\left(g_{1}\right)\right\}\right\} \\
& =\max \left\{\max \left\{w_{1}\left(g_{2}\right), w_{2}\left(g_{2}\right)\right\}, \max \left\{w_{3}\left(g_{1}\right), w_{3}\left(g_{1}\right)\right\}\right\} \\
& =\max \left\{\max \left\{w_{1}\left(g_{2}\right), w_{2}\left(g_{2}\right)\right\}, w_{3}\left(g_{1}\right)\right\} \\
& =\max \left\{\left(w_{1} \cap w_{2}\right)\left(g_{2}\right), w_{3}\left(g_{1}\right)\right\},
\end{aligned}
$$

and so $\left(w_{1} \cap w_{2}\right)\left(g_{1} g_{2} g_{1}^{-1}\right) \leq \max \left\{\left(w_{1} \cap w_{2}\right)\left(g_{2}\right), w_{3}\left(g_{1}\right)\right\}$. Thus $\mu_{1} \cap \mu_{2}=\left(r_{1} \cap r_{2}\right) e^{i\left(w_{1} \cap w_{2}\right)} \bowtie \mu_{3}$.

Corollary 4. Let $I_{n}=\{1,2, \ldots, n\}$ and $\left\{\mu_{i} \mid i \in I_{n}\right\} \subseteq A C F S(G)$ such that $\left\{\mu_{i} \mid i \in I_{n}\right\} \bowtie \xi$. Then $\mu=\cap_{i \in I_{n}} \mu_{i} \bowtie$ $\xi$.

\section{Group homomorphisms and anti complex fuzzy subgroups under $s$-norms}

Definition 10. Let $f: G \rightarrow H$ be a mapping such that $\mu_{G}=r_{G} e^{i w_{G}}$ and $\mu_{H}=r_{H} e^{i w_{H}}$ be two complex fuzzy sets on $G$ and $H$, respectively. Define $f\left(\mu_{G}\right): H \rightarrow[0,1]$ as $f\left(\mu_{G}\right)=f\left(r_{G} e^{i w_{G}}\right)=f\left(r_{G}\right) e^{i f\left(w_{G}\right)}$ such that for all $h \in H$ we define $f\left(r_{G}\right)(h)=\inf \left\{r_{G}(g) \mid g \in G, f(g)=h\right\}$ and $f\left(w_{G}\right)(h)=\inf \left\{w_{G}(g) \mid g \in G, f(g)=h\right\}$. Also define $f^{-1}\left(\mu_{H}\right): G \rightarrow[0,1]$ as $f^{-1}\left(r_{H} e^{i w_{H}}\right)=f^{-1}\left(r_{H}\right) e^{i f^{-1}\left(w_{H}\right)}$ such that for all $g \in G$, we define $f^{-1}\left(r_{H} e^{i w_{H}}\right)(g)=r_{H}(f(g)) e^{i w_{H}(f(g))}$.

Proposition 9. Let $\mu_{G}=r_{G} e^{i w_{G}} \in A C F S(G)$ and $f: G \rightarrow H$ be a group homomorphism, then $f\left(\mu_{G}\right) \in A C F S(H)$.

Proof. (1) Let $h_{1}, h_{2} \in H$ and $g_{1}, g_{2} \in G$ such that $h_{1}=f\left(g_{1}\right)$ and $h_{2}=f\left(g_{2}\right)$. Then

$$
\begin{aligned}
f\left(r_{G}\right)\left(h_{1} h_{2}\right) & =\inf \left\{r_{G}\left(g_{1} g_{2}\right) \mid g_{1}, g_{2} \in G, f\left(g_{1}\right)=h_{1}, f\left(g_{2}\right)=h_{2}\right\} \\
& \leq \inf \left\{S\left(r_{G}\left(g_{1}\right), r_{G}\left(g_{2}\right)\right) \mid g_{1}, g_{2} \in G, f\left(g_{1}\right)=h_{1}, f\left(g_{2}\right)=h_{2}\right\} \\
& =S\left(\inf \left\{r_{G}\left(g_{1}\right) \mid g_{1} \in G, f\left(g_{1}\right)=h_{1}\right\}, \inf \left\{r_{G}\left(g_{2}\right) \mid g_{2} \in G, f\left(g_{2}\right)=h_{2}\right\}\right) \\
& =S\left(f\left(r_{G}\right)\left(h_{1}\right), f\left(r_{G}\right)\left(h_{2}\right)\right),
\end{aligned}
$$

and so $f\left(r_{G}\right)\left(h_{1} h_{2}\right) \leq S\left(f\left(r_{G}\right)\left(h_{1}\right), f\left(r_{G}\right)\left(h_{2}\right)\right)$.

(2) Let $h \in H$ and $g \in G$ such that $h=f(g)$. Then

$$
\begin{aligned}
f\left(r_{G}\right)\left(h^{-1}\right) & =\inf \left\{r_{G}\left(g^{-1}\right) \mid g^{-1} \in G, f\left(g^{-1}\right)=h^{-1}\right\} \\
& \leq \inf \left\{r_{G}(g) \mid g \in G, f^{-1}(g)=h^{-1}\right\} \\
& =\inf \left\{r_{G}(g) \mid g \in G, f(g)=h\right\}=f\left(r_{G}\right)(h),
\end{aligned}
$$

and so $f\left(r_{G}\right)\left(h^{-1}\right) \leq f\left(r_{G}\right)(h)$.

(3) Let $h_{1}, h_{2} \in H$ and $g_{1}, g_{2} \in G$ such that $h_{1}=f\left(g_{1}\right)$ and $h_{2}=f\left(g_{2}\right)$. Then

$$
\begin{aligned}
f\left(w_{G}\right)\left(h_{1} h_{2}\right) & =\inf \left\{w_{G}\left(g_{1} g_{2}\right) \mid g_{1}, g_{2} \in G, f\left(g_{1}\right)=h_{1}, f\left(g_{2}\right)=h_{2}\right\} \\
& \leq \inf \left\{\max \left\{w_{G}\left(g_{1}\right), w_{G}\left(g_{2}\right)\right\} \mid g_{1}, g_{2} \in G, f\left(g_{1}\right)=h_{1}, f\left(g_{2}\right)=h_{2}\right\} \\
& =\max \left\{\inf \left\{w_{G}\left(g_{1}\right) \mid g_{1} \in G, f\left(g_{1}\right)=h_{1}\right\}, \inf \left\{w_{G}\left(g_{2}\right) \mid g_{2} \in G, f\left(g_{2}\right)=h_{2}\right\}\right\} \\
& =\max \left\{f\left(w_{G}\right)\left(h_{1}\right), f\left(w_{G}\right)\left(h_{2}\right)\right\},
\end{aligned}
$$


and thus $f\left(w_{G}\right)\left(h_{1} h_{2}\right) \geq \max \left\{f\left(w_{G}\right)\left(h_{1}\right), f\left(w_{G}\right)\left(h_{2}\right)\right\}$.

(4) Let $h \in H$ and $g \in G$ such that $h=f(g)$. Now

$$
\begin{aligned}
f\left(w_{G}\right)\left(h^{-1}\right) & =\inf \left\{w_{G}\left(g^{-1}\right) \mid g^{-1} \in G, f\left(g^{-1}\right)=h^{-1}\right\} \\
& \geq \inf \left\{w_{G}(g) \mid g^{-1} \in G, f^{-1}(g)=h^{-1}\right\} \\
& =\inf \left\{w_{G}(g) \mid g \in G, f(g)=h\right\}=f\left(w_{G}\right)(h)
\end{aligned}
$$

and therefore $f\left(w_{G}\right)\left(h^{-1}\right) \geq f\left(w_{G}\right)(h)$.

Thus (1) - (4) mean that $f\left(\mu_{G}\right)=f\left(r_{G} e^{i w_{G}}\right)=f\left(r_{G}\right) e^{i f\left(w_{G}\right)} \in \operatorname{ACFS}(H)$.

Proposition 10. Let $\mu_{H}=r_{H} e^{i w_{H}} \in \operatorname{ACFS}(H)$ and $f: G \rightarrow H$ be a group homomorphism, then $f^{-1}\left(\mu_{H}\right) \in$ $\operatorname{ACFS}(G)$.

Proof. (1) Let $g_{1}, g_{2} \in G$, then $f^{-1}\left(r_{H}\right)\left(g_{1} g_{2}\right)=r_{H}\left(f\left(g_{1} g_{2}\right)\right)=r_{H}\left(f\left(g_{1}\right) f\left(g_{2}\right)\right) \leq$ $S\left(r_{H}\left(f\left(g_{1}\right)\right), r_{H}\left(f\left(g_{2}\right)\right)\right)=S\left(f^{-1}\left(r_{H}\right)\left(g_{1}\right), f^{-1}\left(r_{H}\right)\left(g_{2}\right)\right)$, and then $f^{-1}\left(r_{H}\right)\left(g_{1} g_{2}\right) \leq$ $S\left(f^{-1}\left(r_{H}\right)\left(g_{1}\right), f^{-1}\left(r_{H}\right)\left(g_{2}\right)\right)$.

(2) Let $g \in G$, then $f^{-1}\left(r_{H}\right)\left(g^{-1}\right)=r_{H}\left(f\left(g^{-1}\right)\right)=r_{H}\left(f^{-1}(g)\right) \leq r_{H}(f(g))=f^{-1}\left(r_{H}\right)(g)$, and thus $f^{-1}\left(r_{H}\right)\left(g^{-1}\right) \leq f^{-1}\left(r_{H}\right)(g)$.

(3) Let $g_{1}, g_{2} \in G$, so $f^{-1}\left(w_{H}\right)\left(g_{1} g_{2}\right)=w_{H}\left(f\left(g_{1} g_{2}\right)\right)=w_{H}\left(f\left(g_{1}\right) f\left(g_{2}\right)\right) \leq \max \left\{w_{H}\left(f\left(g_{1}\right)\right), w_{H}\left(f\left(g_{2}\right)\right)\right\}=$ $\max \left\{f^{-1}\left(w_{H}\right)\left(g_{1}\right), f^{-1}\left(w_{H}\right)\left(g_{2}\right)\right\}$, and then $\left.f^{-1}\left(w_{H}\right)\left(g_{1} g_{2}\right) \leq \max \left\{f^{-1}\left(w_{H}\right)\left(g_{1}\right), f^{-1}\left(w_{H}\right)\left(g_{2}\right)\right)\right\}$.

(4) Let $g \in G$, then $f^{-1}\left(w_{H}\right)\left(g^{-1}\right)=w_{H}\left(f^{-1}(g)\right) \leq w_{H}(f(g))=f^{-1}\left(w_{H}\right)(g)$ and then $f^{-1}\left(w_{H}\right)\left(g^{-1}\right) \leq$ $f^{-1}\left(w_{H}\right)(g)$.

Therefore (1)-(4) give us $f^{-1}\left(r_{H} e^{i w_{H}}\right)(g)=r_{H}(f(g)) e^{i w_{H}(f(g))} \in \operatorname{ACFS}(G)$.

Proposition 11. Let $\mu_{G}=r_{G} e^{i w_{G}} \in \operatorname{NACFS}(G)$ and $f: G \rightarrow H$ be a group homomorphism. Then $f\left(\mu_{G}\right) \in$ $\operatorname{NACFS}(H)$.

Proof. From Proposition 9, we have $f\left(\mu_{G}\right) \in A C F S(H)$. Let $g_{1}, g_{2} \in G$ and $h_{1}, h_{2} \in H$ such that $f\left(g_{1}\right)=h_{1}$ and $f\left(g_{2}\right)=h_{2}$. Now

$$
\begin{aligned}
f\left(r_{G}\right)\left(h_{1} h_{2} h_{1}^{-1}\right) & =\inf \left\{r_{G}\left(g_{1} g_{2} g_{1}^{-1}\right) \mid f\left(g_{1} g_{2} g_{1}^{-1}\right)=h_{1} h_{2} h_{1}^{-1}\right\} \\
& =\inf \left\{r_{G}\left(g_{2}\right) \mid f\left(g_{1}\right) f\left(g_{2}\right) f\left(g_{1}^{-1}\right)=h_{1} h_{2} h_{1}^{-1}\right\} \\
& =\inf \left\{r_{G}\left(g_{2}\right) \mid f\left(g_{1}\right) f\left(g_{2}\right) f^{-1}\left(g_{1}\right)=h_{1} h_{2} h_{1}^{-1}\right\} \\
& =\inf \left\{r_{G}\left(g_{2}\right) \mid f\left(g_{2}\right)=h_{2}\right\}=f\left(r_{G}\right)\left(h_{2}\right) .
\end{aligned}
$$

Also

$$
\begin{aligned}
f\left(w_{G}\right)\left(h_{1} h_{2} h_{1}^{-1}\right) & =\inf \left\{w_{G}\left(g_{1} g_{2} g_{1}^{-1}\right) \mid f\left(g_{1} g_{2} g_{1}^{-1}\right)=h_{1} h_{2} h_{1}^{-1}\right\} \\
& =\inf \left\{w_{G}\left(g_{2}\right) \mid f\left(g_{1}\right) f\left(g_{2}\right) f\left(g_{1}^{-1}\right)=h_{1} h_{2} h_{1}^{-1}\right\} \\
& =\inf \left\{w_{G}\left(g_{2}\right) \mid f\left(g_{1}\right) f\left(g_{2}\right) f^{-1}\left(g_{1}\right)=h_{1} h_{2} h_{1}^{-1}\right\} \\
& =\inf \left\{w_{G}\left(g_{2}\right) \mid f\left(g_{2}\right)=h_{2}\right\}=f\left(w_{G}\right)\left(h_{2}\right) .
\end{aligned}
$$

Then $f\left(\mu_{G}\right)\left(h_{1} h_{2} h_{1}^{-1}\right)=f\left(r_{G}\right)\left(h_{1} h_{2} h_{1}^{-1}\right) e^{i f\left(w_{G}\right)\left(h_{1} h_{2} h_{1}^{-1}\right)}=f\left(r_{G}\right)\left(h_{2}\right) e^{i f\left(w_{G}\right)\left(h_{2}\right)}=f\left(\mu_{G}\right)\left(h_{2}\right)$. and so $f\left(\mu_{G}\right) \in \operatorname{NACFS}(H)$.

Proposition 12. Let $\mu_{H}=r_{H} e^{i w_{H}} \in \operatorname{NACFS}(H)$ and $f: G \rightarrow H$ be a group homomorphism, then $f^{-1}\left(\mu_{H}\right) \in$ $N A C F S(G)$.

Proof. Using Proposition 10, we get $f^{-1}\left(\mu_{H}\right) \in A C F S(G)$. Let $g_{1}, g_{2} \in G$, then $f^{-1}\left(r_{H}\right)\left(g_{1} g_{2} g_{1}^{-1}\right)=$ $r_{H}\left(f\left(g_{1} g_{2} g_{1}^{-1}\right)\right)=r_{H}\left(f\left(g_{1}\right) f\left(g_{2}\right) f\left(g_{1}^{-1}\right)\right)=r_{H}\left(f\left(g_{1}\right) f\left(g_{2}\right) f^{-1}\left(g_{1}\right)\right)=r_{H}\left(f\left(g_{2}\right)\right)=f^{-1}\left(r_{H}\right)\left(g_{2}\right)$. Also $f^{-1}\left(w_{H}\right)\left(g_{1} g_{2} g_{1}^{-1}\right)=w_{H}\left(f\left(g_{1} g_{2} g_{1}^{-1}\right)\right)=w_{H}\left(f\left(g_{1}\right) f\left(g_{2}\right) f\left(g_{1}^{-1}\right)\right)=w_{H}\left(f\left(g_{1}\right) f\left(g_{2}\right) f^{-1}\left(g_{1}\right)\right)=w_{H}\left(f\left(g_{2}\right)\right)=$ $f^{-1}\left(w_{H}\right)\left(g_{2}\right)$. Thus $f^{-1}\left(\mu_{H}\right)\left(g_{1} g_{2} g_{1}^{-1}\right)=f^{-1}\left(r_{H}\right)\left(g_{1} g_{2} g_{1}^{-1}\right) e^{i f^{-1}\left(w_{H}\right)\left(g_{1} g_{2} g_{1}^{-1}\right)}=f^{-1}\left(r_{H}\right)\left(g_{2}\right) e^{i f^{-1}\left(w_{H}\right)\left(g_{2}\right)}=$ $f^{-1}\left(\mu_{H}\right)\left(g_{2}\right)$ and thus $f^{-1}\left(\mu_{H}\right) \in \operatorname{NACFS}(G)$. 
Proposition 13. Let $\mu_{1}=r_{1} e^{i w_{1}} \in \operatorname{ACFS}(G)$ and $\mu_{2}=r_{2} e^{i w_{2}} \in \operatorname{ACFS}(G)$ and $f: G \rightarrow H$ be a group homomorphism. If $\mu_{1} \bowtie \mu_{2}$, then $f\left(\mu_{1}\right) \bowtie f\left(\mu_{2}\right)$.

Proof. We know that $f\left(\mu_{1}\right)=f\left(r_{1}\right) e^{i f\left(w_{1}\right)}$ and $f\left(\mu_{2}\right)=f\left(r_{2}\right) e^{i f\left(w_{2}\right)}$. By Proposition 9, we have $f\left(\mu_{1}\right) \in$ $\operatorname{ACFS}(H)$ and $f\left(\mu_{2}\right) \in \operatorname{ACFS}(H)$. Let $g_{1}, g_{2} \in G$ and $h_{1}, h_{2} \in H$ such that $f\left(g_{1}\right)=h_{1}$ and $f\left(g_{2}\right)=h_{2}$. Since $\mu_{1} \bowtie \mu_{2}$ so $r_{1}\left(g_{1} g_{2} g_{1}^{-1}\right) \leq S\left(r_{1}\left(g_{2}\right), r_{2}\left(g_{1}\right)\right)$ and $w_{1}\left(g_{1} g_{2} g_{1}^{-1}\right) \leq \max \left\{w_{1}\left(g_{2}\right), w_{2}\left(g_{1}\right)\right\}$. Now

$$
\begin{aligned}
f\left(r_{1}\right)\left(h_{1} h_{2} h_{1}^{-1}\right) & =\inf \left\{r_{1}\left(g_{1} g_{2} g_{1}^{-1}\right) \mid f\left(g_{1} g_{2} g_{1}^{-1}\right)=h_{1} h_{2} h_{1}^{-1}\right\} \\
& \leq \inf \left\{S\left(r_{1}\left(g_{2}\right), r_{2}\left(g_{1}\right)\right) \mid f\left(g_{1}\right) f\left(g_{2}\right) f\left(g_{1}^{-1}\right)=h_{1} h_{2} h_{1}^{-1}\right\} \\
& =\inf \left\{T\left(r_{1}\left(g_{2}\right), r_{2}\left(g_{1}\right)\right) \mid f\left(g_{1}\right) f\left(g_{2}\right) f^{-1}\left(g_{1}\right)=h_{1} h_{2} h_{1}^{-1}\right\} \\
& =S\left(\inf \left\{r_{1}\left(g_{2}\right) \mid f\left(g_{2}\right)=h_{2}\right\}, \inf \left\{r_{2}\left(g_{1}\right) \mid f\left(g_{1}\right)=h_{1}\right\}\right) \\
& =S\left(f\left(r_{1}\right)\left(h_{2}\right), f\left(r_{2}\right)\left(h_{1}\right)\right),
\end{aligned}
$$

and then $f\left(r_{1}\right)\left(h_{1} h_{2} h_{1}^{-1}\right) \leq S\left(f\left(r_{1}\right)\left(h_{2}\right), f\left(r_{2}\right)\left(h_{1}\right)\right)$. Also

$$
\begin{aligned}
f\left(w_{1}\right)\left(h_{1} h_{2} h_{1}^{-1}\right) & =\inf \left\{w_{1}\left(g_{1} g_{2} g_{1}^{-1}\right) \mid f\left(g_{1} g_{2} g_{1}^{-1}\right)=h_{1} h_{2} h_{1}^{-1}\right\} \\
& \leq \inf \left\{\max \left\{w_{1}\left(g_{2}\right), w_{2}\left(g_{1}\right)\right\} \mid f\left(g_{1}\right) f\left(g_{2}\right) f\left(g_{1}^{-1}\right)=h_{1} h_{2} h_{1}^{-1}\right\} \\
& =\inf \left\{\max \left\{w_{1}\left(g_{2}\right), w_{2}\left(g_{1}\right)\right\} \mid f\left(g_{1}\right) f\left(g_{2}\right) f^{-1}\left(g_{1}\right)=h_{1} h_{2} h_{1}^{-1}\right\} \\
& =\max \left\{\inf \left\{w_{1}\left(g_{2}\right) \mid f\left(g_{2}\right)=h_{2}\right\}, \inf \left\{w_{2}\left(g_{1}\right) \mid f\left(g_{1}\right)=h_{1}\right\}\right\} \\
& =\max \left\{f\left(w_{1}\right)\left(h_{2}\right), f\left(w_{2}\right)\left(h_{1}\right)\right\},
\end{aligned}
$$

and so $f\left(w_{1}\right)\left(h_{1} h_{2} h_{1}^{-1}\right) \leq \max \left\{f\left(w_{1}\right)\left(h_{2}\right), f\left(w_{2}\right)\left(h_{1}\right)\right\}$. Hence $f\left(\mu_{1}\right) \bowtie f\left(\mu_{2}\right)$.

Proposition 14. Let $\mu_{1}=r_{1} e^{i w_{1}} \in A C F S(H), \quad \mu_{2}=r_{2} e^{i w_{2}} \in A C F S(H)$ and $f: G \rightarrow H$ be a group homomorphism. If $\mu_{1} \bowtie \mu_{2}$, then $f^{-1}\left(\mu_{1}\right) \bowtie f^{-1}\left(\mu_{2}\right)$.

Proof. Let $f^{-1}\left(\mu_{1}\right)=f^{-1}\left(r_{1}\right) e^{i f^{-1}\left(w_{1}\right)}$ and $f^{-1}\left(\mu_{2}\right)=f^{-1}\left(r_{2}\right) e^{i f^{-1}\left(w_{2}\right)}$. From Proposition 10, we obtain $f^{-1}\left(\mu_{1}\right) \in \operatorname{ACFS}(G)$ and $f^{-1}\left(\mu_{2}\right) \in \operatorname{ACFS}(G)$. Let $g_{1}, g_{2} \in G$, then

$$
\begin{aligned}
f^{-1}\left(r_{1}\right)\left(g_{1} g_{2} g_{1}^{-1}\right) & =r_{1}\left(f\left(g_{1} g_{2} g_{1}^{-1}\right)\right)=r_{1}\left(f\left(g_{1}\right) f\left(g_{2}\right) f\left(g_{1}^{-1}\right)\right)=r_{1}\left(f\left(g_{1}\right) f\left(g_{2}\right) f^{-1}\left(g_{1}\right)\right) \\
& \leq S\left(r_{1}\left(f\left(g_{2}\right)\right), r_{2}\left(f\left(g_{1}\right)\right)\right)=S\left(f^{-1}\left(r_{1}\right)\left(g_{2}\right), f^{-1}\left(r_{2}\right)\left(g_{1}\right) .\right.
\end{aligned}
$$

Also

$$
\begin{aligned}
f^{-1}\left(w_{1}\right)\left(g_{1} g_{2} g_{1}^{-1}\right) & =w_{1}\left(f\left(g_{1} g_{2} g_{1}^{-1}\right)\right)=w_{1}\left(f\left(g_{1}\right) f\left(g_{2}\right) f\left(g_{1}^{-1}\right)\right)=w_{1}\left(f\left(g_{1}\right) f\left(g_{2}\right) f^{-1}\left(g_{1}\right)\right) \\
& \leq \max \left\{w_{1}\left(f\left(g_{2}\right)\right), w_{2}\left(f\left(g_{1}\right)\right)\right\}=\max \left\{f^{-1}\left(w_{1}\right)\left(g_{2}\right), f^{-1}\left(w_{2}\right)\left(g_{1}\right\} .\right.
\end{aligned}
$$

Therefore $f^{-1}\left(\mu_{1}\right) \bowtie f^{-1}\left(\mu_{2}\right)$.

Acknowledgments: I would like to thank the referees for carefully reading the manuscript and making several helpful comments to increase the quality of the paper.

Conflicts of Interest: "The author declares no conflict of interest."

\section{References}

[1] Zadeh, L. A. (1965). Fuzzy sets. Information and Control, 8(3), 338-353.

[2] Rosenfeld, A. (1971). Fuzzy groups. Journal of Mathematical Analysis and Applications, 35(3), 512-517.

[3] Mordeson, J. N., Bhutani, K. R., \& Rosenfeld, A. (2005). Fuzzy Group Theory (Vol. 182). New York: Springer.

[4] Mukherjee, N. P., \& Bhattacharya, P. (1984). Fuzzy normal subgroups and fuzzy cosets. Information Sciences, 34(3), 225-239.

[5] Anthony, J. M., \& Sherwood, H. (1982). A characterization of fuzzy subgroups. Fuzzy Sets and Systems, 7(3), $297-305$.

[6] Sessa, S. (1984). On fuzzy subgroups and fuzzy ideals under triangular norms. Fuzzy Sets and Systems, 13(1), 95-100.

[7] Osman, M. A. (1987). On some product of fuzzy subgroups. Fuzzy Sets and Systems, 24(1), 79-86. 
[8] Alsarahead, M. O., \& Ahmad, A. G. (2017). Complex fuzzy subgroups. Applied Mathematical Sciences, 11(41), 2011-2021.

[9] Rasuli, R. (2016). Fuzzy Ideals of Subtraction Semigroups with Respect to A t-norm and A t-conorm. The Journal of Fuzzy Mathematics Los Angeles, 24(4), 881-892.

[10] Rasuli, R. (2016). Fuzzy modules over a t-norm. International Journal of Open Problems in Computer Science and Mathematics, 238(4004), 1-7.

[11] Rasuli, R. (2016). Fuzzy Subrings over a t-norm. The Journal of Fuzzy Mathematics Los Angeles, 24(4), 995-1000.

[12] Algebra, T. H. (1974). Springer Graduate texts in Mathematics. Springer-Verlag, New York.

[13] Rasuli, R. (2016). Fuzzy modules over a t-norm. International Journal of Open Problems in Computer Science and Mathematics, 238(4004), 1-7.

[14] Ramot, D., Friedman, M., Langholz, G., \& Kandel, A. (2003). Complex fuzzy logic. IEEE Transactions on Fuzzy Systems, 11(4), 450-461.

[15] Buckley, J. J., \& Eslami, E. (2002). An introduction to fuzzy logic and fuzzy sets (Vol. 13). Springer Science \& Business Media.

(C) 2020 by the authors; licensee PSRP, Lahore, Pakistan. This article is an open access article distributed under the terms and conditions of the Creative Commons Attribution (CC-BY) license (http://creativecommons.org/licenses/by/4.0/). 